\title{
Uso de crack por filhos de mães usuárias dessa droga: revisão sistemática
}

\section{Crack use by children of mothers whom use this drug: a systematic review}

DOI: $10.46919 / \operatorname{archv} 1 \mathrm{n} 5-013$

Recebimento dos originais: 10/07/2020

Aceitação para publicação: 30/08/2020

\author{
Bárbara Borges D'Angelo \\ Médica pela Universidade UNIFENAS-BH, \\ e-mail: dra.barbarabdrocha@gmail.com \\ Ana Carolina Barreto Martyn Costa \\ Médica pela universidade UNIFENAS-BH, \\ e-mail: anacmartyn@gmail.com \\ Ana Clara de Moraes Bittencourt \\ Médica pela universidade UNIFENAS-BH, \\ e-mail: anaclaradmb@gmail.com

\section{Camila de Oliveira} \\ Média pela universidade UNIFENAS-BH, \\ e-mail: camilinhazsc@hotmail.com

\section{Caroline Siriano Bonagura} \\ Médica pela universidade UNIFENAS-BH, \\ e-mail: caroline_bonagura@hotmail.com \\ Nathan Mendes Souza \\ Orientador e Professor do Curso de Medicina da UNIFENAS-BH, \\ e-mail: nathanmendes@ hotmail.com
}

\section{RESUMO}

Objetivo: Revisar o conhecimento científico publicado sobre o uso de crack por filhos de mães que usaram/usam crack. Métodos: Revisão sistemática realizada nas bases de dados do Portal BVS, SciELO, Science Direct, Portal Periódicos CAPES e Google Acadêmico para encontrar artigos advindos das referências daqueles já incluídos. Os estudos foram avaliados por meio de ferramentas metodológicas reconhecidas. Resultados: Encontrou-se como fator de risco direto para o uso de crack por filhos de dependentes a influência familiar e social. Além disso, outros fatores de risco também foram comtemplados como a exposição pré-natal e a herdabilidade de vícios. Todavia, também foram abordados fatores protetores, sendo eles disponibilidade de informações, estrutura familiar protetora, a desaprovação do meio social ao uso da droga, o interesse familiar na vida da criança/adolescente e atividades socioeducativas. Conclusão: Não foi encontrada relação substancial entre o uso de crack durante a gestação por mães usuárias de crack e o futuro uso de seus filhos. Porém, foi constatado que a exposição pós-natal, por meio da influência familiar e social, é o principal fator de risco para o uso e abuso de drogas, especificamente o crack, principalmente se existir a convivência intima com um familiar usuário.

Palavras-chave: Crack cocaine, mothers, child, drug users, epidemiology. 


\section{ABSTRACT}

Objective: To review the published scientific knowledge about the use of crack by children of mothers who used / use crack. Methods: Systematic review carried out in the databases of the VHL Portal, SciELO, Science Direct, CAPES Periodical Portal and Google Scholar to find articles from the references of those already included. The studies were evaluated using recognized methodological tools. Results: Family and social influence was found as a direct risk factor for the use of crack by children of dependents. In addition, other risk factors have also been addressed, such as prenatal exposure and the heritability of addictions. However, protective factors were also addressed, such as availability of information, protective family structure, disapproval of the social environment for drug use, family interest in the life of the child / adolescent and socio-educational activities. Conclusion: There was no substantial relationship between the use of crack during pregnancy by mothers who use crack and the future use of their children. However, it was found that postnatal exposure, through family and social influence, is the main risk factor for drug use and abuse, specifically crack, especially if there is close contact with a family member.

Keywords: Crack cocaine, mothers, child, drug users, epidemiology.

\section{INTRODUÇÃO}

O crack é uma pedra formada da mistura da pasta básica da cocaína com bicarbonato de sódio sendo utilizado mundialmente como potente estimulador do Sistema Nervoso Central. Essa droga surgiu no Brasil no final da década de 1980 e por seus efeitos estimulantes e prazerosos, aliados aos baixos custos da comercialização ilegal, seu consumo se alastrou pelo território brasileiro. Em 1999, o Instituto Brasileiro de Geografia e Estatística (IBGE) estimou em 1,2 milhões de brasileiros usuários dessa droga ${ }^{1}$. No entanto, no recente estudo divulgado pela Fundação Oswaldo Cruz (Fiocruz) o número de usuários regulares de crack e/ou de formas similares de cocaína fumada (merla, pasta-base e oxi) nas capitais brasileiras somam 370 mil pessoas, representando 35\% do total de consumidores de drogas ilícitas. Tal levantamento mostra ainda que a maioria desses usuários é composta de adultos-jovens com idade média de 30 anos, e ainda destaca que 14\% dessa população usuária de crack são menores de idade².

Dessa população de consumidores de crack há o subgrupo das mulheres usuárias dessa droga (crack) que continuam o consumo durante a gestação. De acordo a pesquisa da Fiocruz divulgada esse ano (2013), das mulheres que participaram do referente estudo, cerca de 10\% delas relataram estar grávidas no momento da entrevista ${ }^{2}$. Tal fato, ainda é corroborado pela Organização Mundial da Saúde (OMS), ao afirmar que o número de usuários da droga vem aumentando ano após ano, inclusive de gestantes, tendo assim um impacto significativo para a saúde pública.

Sabe-se que o uso da droga durante a gravidez pode desencadear abortos espontâneos, prematuridade, diminuição do crescimento do feto e outras alterações perinatais. Além disso, aqueles bebês que nascem vivos podem apresentar retardo mental ou outros distúrbios comportamentais ${ }^{3}$. 
Segundo Fliglie ${ }^{4}$, filhos de pessoas com dependência química apresentam risco aumentado para o desenvolvimento de transtornos psiquiátricos e para o consumo de substâncias psicoativas. No entanto, este estudo não compara o uso de drogas durante a gestação com um risco maior de dependência futura na descendência dessas gestantes.

Estudos demonstram que há uma forte associação entre antecedentes familiares de uso de drogas com o abuso de drogas tanto na adolescência quanto na vida adulta ${ }^{5}$. Lam $^{6}$ e Rudolph ${ }^{7}$ corroboram esta associação ao afirmarem que o ambiente familiar influencia diretamente na probabilidade de crianças filhas de usuários de drogas se tornarem também futuras usuárias. Assim, crianças que moram com pais usuários de substâncias ilícitas constituem um grupo de alto risco e de se tornarem dependentes de drogas $^{6}$. A preocupação com filhos de dependentes químicos vem notoriamente ocupando maior atenção na área de saúde, e investir nessa população significa trabalhar com a prevenção eletiva, por ser dirigida a um determinado grupo de risco ${ }^{5}$.

Contudo há escassez na literatura científica referente a relação entre o uso de substâncias ilícitas pelas mães e o aumento da probabilidade dos filhos se tornarem usuários dessas substâncias, principalmente do crack. O objetivo deste estudo é revisar o conhecimento científico publicado sobre o uso de crack por filhos de mães que usaram/usam crack.

\section{METODOLOGIA}

Realizou-se uma revisão sistemática da literatura científica sobre os efeitos do uso de crack durante a gestação, especialmente no uso futuro dessa droga por filhos de mães dependentes. De acordo com o modelo PICOT, buscou-se por estudos cuja população estudada foram mães que tenham usado crack em qualquer fase durante os ciclos gravídico-puerperal; e cujas filhos tornaram-se usuários futuramente, comparando-se com os filhos que não tornaram-se usuários nas mesmas condições.

Os descritores MeSH e DeCS e termos correlatos utilizados na construção das estratégias de busca estão descritos na Tabela 1.

Tabela 1. Descritores Mesh e DeCS e termos correlatos.

\begin{tabular}{|c|c|c|}
\hline Descritores MeSH & Descritores DeCs & Termos correlatos \\
\hline Crack cocaine & Crack; cocaine; cocaine; crack cocaine & Cocaína \\
\hline Mothers & Mothers; madres; mães & Crianças \\
\hline Child & Child; nino; crianças & \\
\hline Drug users & Drug users; consumidores de drogas; & \\
& Usuarios de drogas & \\
\hline & $\begin{array}{c}\text { Cocaine-related disorders; transtornos } \\
\text { relacionados con Cocaína; transtornos } \\
\text { relacionados ao uso de cocaína }\end{array}$ & \\
\hline & Epidemiology; epidemiologia; & \\
& epidemiologia & \\
\hline
\end{tabular}




\begin{tabular}{|c|c|c|}
\hline Gravidity & $\begin{array}{c}\text { Pregnant women; mujeres embarazadas; } \\
\text { gestantes }\end{array}$ & \\
\hline
\end{tabular}

Buscou-se estudos publicados no Portal BVS, SciELO, Science Direct, Portal Periódicos CAPES, Google acadêmico e nas referências dos estudos incluídos. Não houve restrição de idiomas e usou-se os limites: estudos indexados até setembro de 2013, humanos e estudos empíricos. As estratégias de busca utilizadas em cada base de dados pesquisadas e os artigos recuperados estão descritas na Tabela 2. Buscou-se ainda documentos não publicados em revistas científicas, mas que constam nos portais IDSUS e IBGE.

Utilizou-se os seguintes critérios de inclusão: estudos empíricos de todos os tipos publicados desde o início de disponibilidade de cada base de dados pesquisada até setembro de 2013, cujo foco do artigo seja o uso de crack em gestantes, puérperas ou lactentes e por seus filhos. Os critérios de exclusão utilizados foram: artigos cujo foco fosse sobre síndrome da abstinência neonatal; uso de crack na gestação e más formações congênitas; gestantes dependentes de crack e outras drogas; uso de outras drogas que não crack por crianças filhas de mães dependentes de crack; temas não relacionados diretamente ou indiretamente ao crack. A aplicação dos critérios de inclusão e exclusão foi feita por pares, sendo que não houve discrepância entre estes. O total de artigos pesquisados, incluídos e excluídos estão demonstrados na Figura 1.

Tabela 2. Estratégias de busca e bases de dados pesquisadas.

\begin{tabular}{|c|c|c|c|}
\hline Estratégia de Busca & $\begin{array}{c}\text { CAPES } \\
\text { Periódicos }\end{array}$ & SciELQ & BVS \\
\hline $\begin{array}{l}\text { 1. Crack cocaine AND children of substance } \\
\text { users AND NOT heroin AND NOT African } \\
\text { americans AND NOT HIV AND NOT } \\
\text { Substance abuse treatment AND NOT juvenile } \\
\text { drug abuse }\end{array}$ & 325 & - & - \\
\hline $\begin{array}{l}\text { 2. Crack cocaine AND children of substance } \\
\text { users AND NOT juvenile drug abuse }\end{array}$ & 197 & - & - \\
\hline 3. Crack AND children & - & 5 & - \\
\hline $\begin{array}{l}\text { 4. (crack cocaine) and children AND LIMIT- } \\
\text { TO (topics, "cocaine, drug abuse, cocaine } \\
\text { exposure, pre natal, child, woman") }\end{array}$ & 418 & - & - \\
\hline 5. crack AND mothers & - & - & 44 \\
\hline 6. crack AND children & - & - & 7 \\
\hline 7. (crack cocaine) AND children & - & - & 302 \\
\hline $\begin{array}{l}\text { 8. (crack OR crack çcaine OR cocaina) AND } \\
\text { (gravidez OR gravida OR gestante OR } \\
\text { pregnancx OR pregnant OR embaraza OR } \\
\text { embarazada) AND (crianca OR child* OR nino } \\
\text { OR neonato OR nelvborn) AND (dependencia } \\
\text { OR dependence) AND mi:("Transtornos } \\
\text { Relacionados ao Uso de Substâncias" OR } \\
\text { "Efeitos Tardios da Exposição Pré-Natal" OR } \\
\text { "Transtornos Relacionados ao Uso de Cocaina" } \\
\text { OR "Resultado da Gravidez" OR "Deficiências } \\
\text { do Desenvolvimento" OR "Drogas Ilicitas") } \\
\text { AND (usuarie OR ysuarios OR ysers) }\end{array}$ & - & - & 71 \\
\hline
\end{tabular}


Figura 1. Fluxograma com o número absoluto de artigos incluídos e excluídos do estudo (Parte 1).

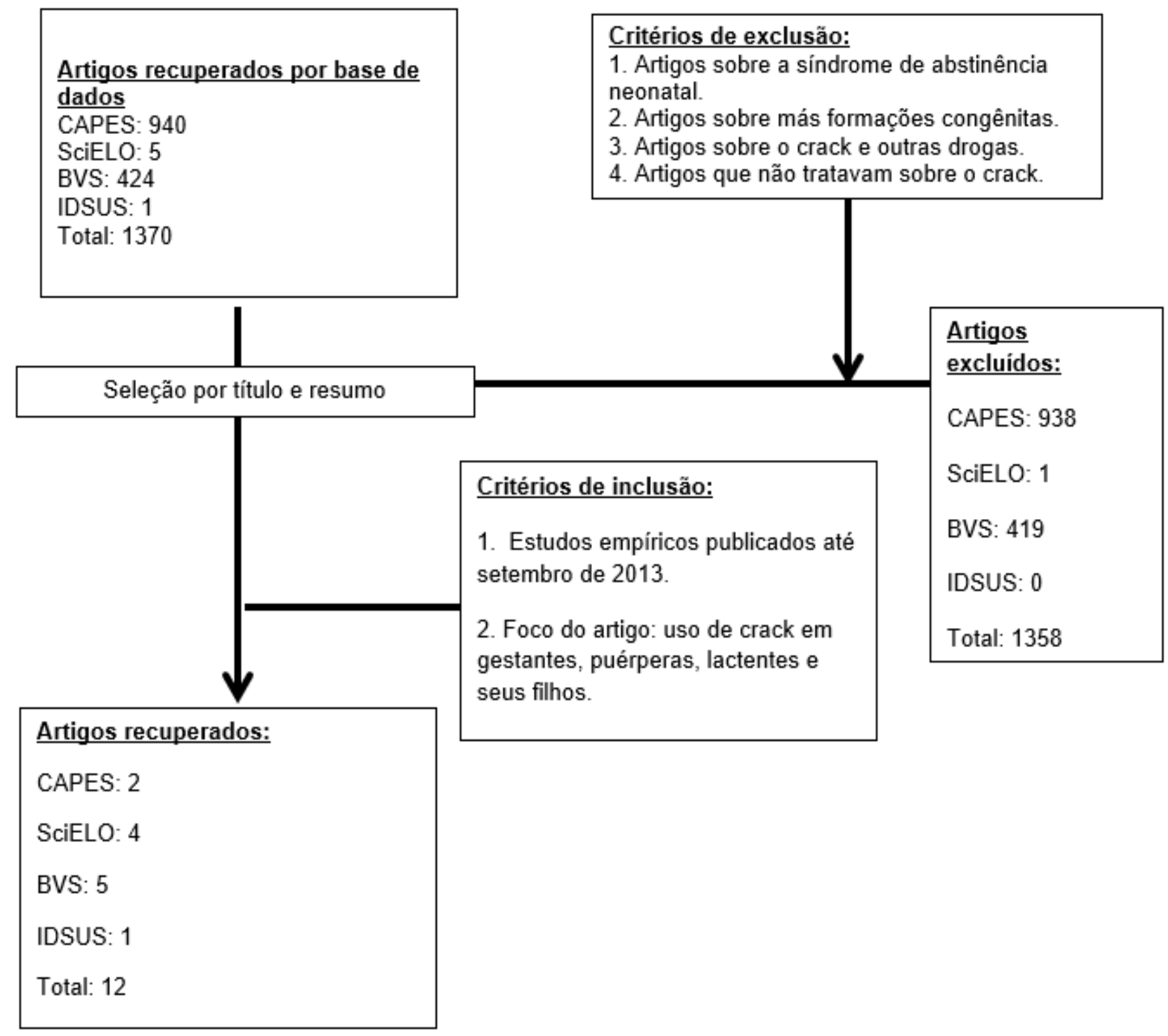


Figura 1. Fluxograma com o número absoluto de artigos incluídos e excluídos do estudo (Parte 2).

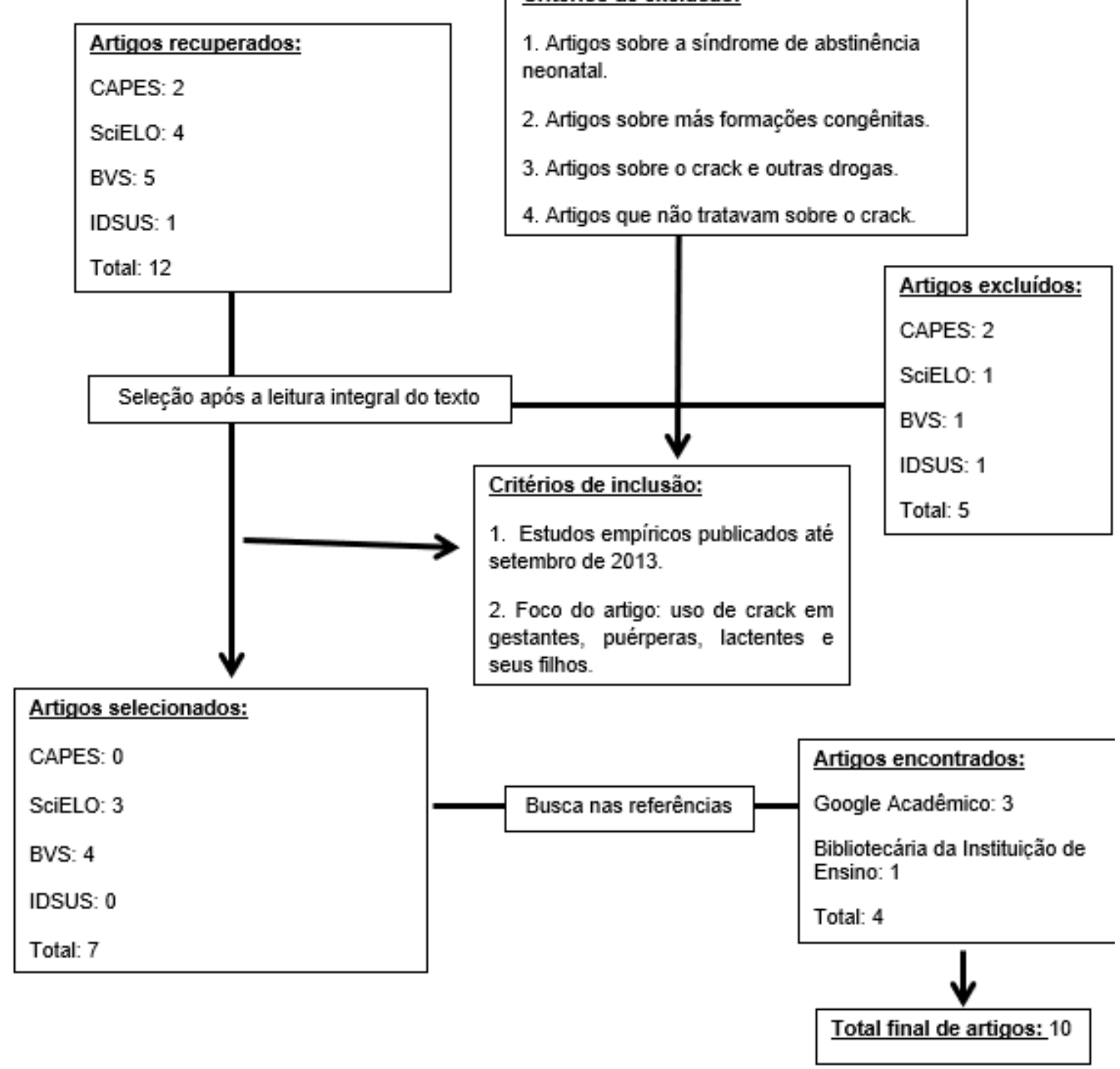

\section{RESULTADOS}

Recuperou-se 1370 artigos, sendo que 10 contemplaram os critérios de inclusão (Figura 1). Observou-se grande variedade de tipos de estudo, intervenções e desfecho. Dos 10, estudos incluídos, 2 eram do tipo qualitativo, 1 do tipo qualitativo descritivo, 2 do tipo revisão sistemática, 2 do tipo transversal, 1 do tipo coorte prospectivo e 1 do tipo longitudinal qualitativo. Desses artigos, 4 foram publicados em inglês e 6 em português. Sendo que, 4 foram desenvolvidos nos EUA e 6 no Brasil. Observou-se que em geral foram feitas entrevistas semiestruturadas com populações de usuários de crack - adolescentes e jovens adultos e suas famílias. Não obteve-se estudos com idosos usuários de crack filhos de mães usuárias de crack. A característica de cada artigo pode ser melhor observada na tabela 3.

Os estudos de Swadi ${ }^{8}$, Fliglie ${ }^{4}$, Oliveira $^{9}$, Rudolph $^{7}$, Santos $^{10}$ e Seleghim ${ }^{5}$, publicados, respectivamente, nos periódicos Drug and Acohol Dependence, Revista de Psiquiatria Clínica, Revista 
Eletrônica de Saúde Mental e Álcool e Drogas, Drug and Acohol Dependence, IV Jornada de Pesquisa em Psicologia da UNISC- Universidade Santa Cruz do Sul e Acta Paulista de Enfermagem investigaram os fatores que influenciam o uso de crack e que estão presentes no ambiente familiar. Eles concluem que a presença de cultura implícita do consumo de drogas e a existência de mais de um familiar que usou drogas está associado ao desenvolvimento de distúrbios sociais e psiquiátricos e à grande probabilidade de usar drogas e, mais especificamente, o uso/fumo do crack em seus ambientes na fase adulta, aumentando também a dependência dessas.

Já os artigos de $\mathrm{Swadi}^{8}$, Zila ${ }^{11}$, Lam $^{6}$, Oliveira ${ }^{9}$ publicados, respectivamente, nos periódicos Revista de Saúde Pública, Drug and Acohol, Dependence, Journal of Pediatric Psychology e Revista Eletrônica de Saúde Mental e Álcool e Drogas também reforçam a correlação entre o ambiente familiar e o uso de crack, tanto como fator de risco como fator protetor. Eles concluíram que os fatores protetores são a disponibilidade de informações, estrutura familiar protetora, a desaprovação do meio social ao uso da droga, o interesse familiar na vida da criança/adolescente e atividades socioeducativas (atividades esportivas, cursos profissionalizantes, incentivo ao potencial criativo).

O estudo de Delaney-Black ${ }^{12}$ publicado na revista Neurotoxicology and Teratology identificou que tanto a exposição pré-natal quanto a exposição pós-natal à cocaína (uso da droga pelos pais ou cuidadores) foram unicamente relacionados ao uso de cocaína por adolescentes e também foi encontrada relação indireta entre o uso da droga e a exposição a essa na comunidade. Por fim, o artigo de Marques ${ }^{13}$ publicado na Revista da Associação Médica Brasileira, observa que existe transmissão familiar de dependência de cocaína e herdabilidade moderada a alta para a maioria dos vícios.

Em praticamente todos os artigos observa-se que a família e o ambiente familiar em torno do indivíduo, são tidos como uma das principais formas de influência para o consumo ou não do crack e outras drogas, seja de forma direta ou indireta (Gráfico 1). Em alguns estudos há a evidência de que a relação com a mãe, especificamente, pode ser considerada tanto fator protetor quanto de risco. 
Gráfico 1. Número absoluto de artigos encontrados relacionando os fatores de risco para o uso de drogas por crianças/jovens adultos.

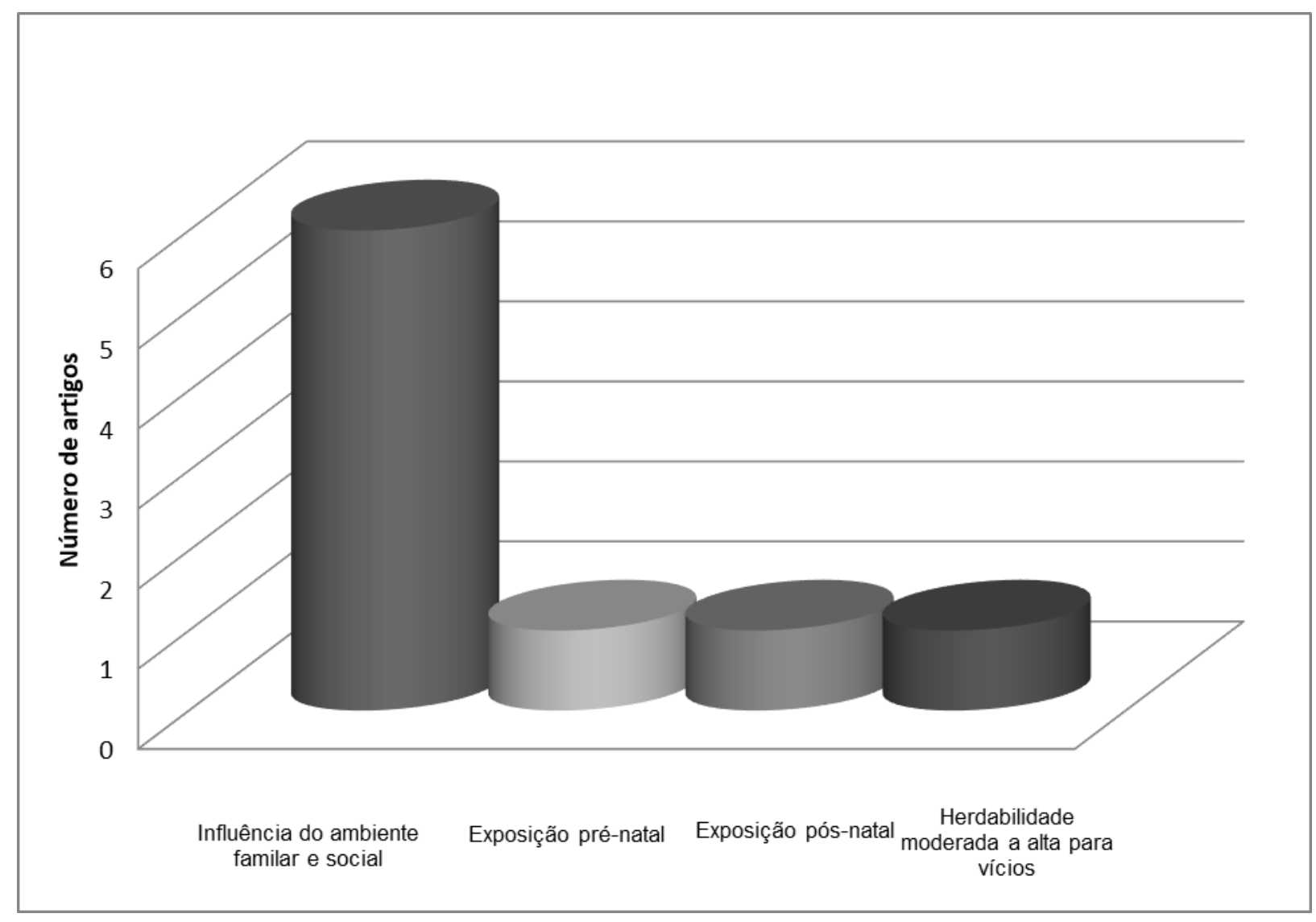


Tabela 3. Características dos artigos incluídos

\begin{tabular}{|c|c|c|c|c|c|}
\hline 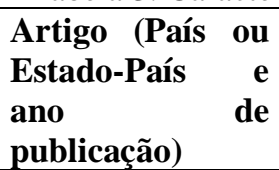 & Participantes & Local & $\begin{array}{ll}\text { Tipo } & \text { e } \\
\text { qualidade } & \\
\text { metodológica } & \\
\text { dos estudos } & \\
\end{array}$ & Objetivos & Resultados \\
\hline $\begin{array}{l}\text { Influência do } \\
\text { ambiente familiar } \\
\text { no consumo de } \\
\text { crack em usuários } \\
(\text { Paraná-Brasil, } \\
2013)^{5}\end{array}$ & $\begin{array}{l}15 \text { famílias de } \\
\text { usuários de crack } \\
\text { (sete mães, cinco } \\
\text { irmãos, dois pais } \\
\text { e uma tia) em } \\
\text { tratamento em um } \\
\text { serviço } \\
\text { especializado, por } \\
\text { meio de entrevista } \\
\text { semiestruturada }\end{array}$ & 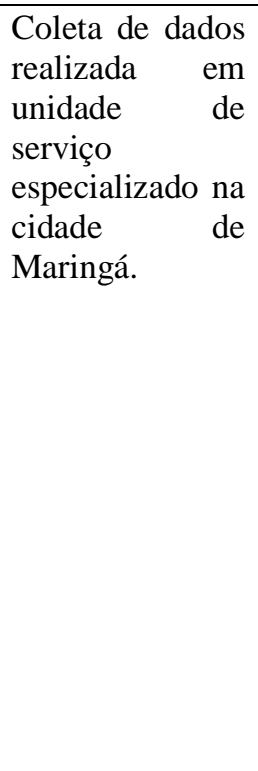 & $\begin{array}{l}\text { Qualitativo } \\
\text { (7/10 CASP } \\
\text { Qualitative } \\
\text { Research } \\
\text { Checklist) }\end{array}$ & $\begin{array}{l}\text { Analisar a influência } \\
\text { do ambiente familiar } \\
\text { no consumo de crack } \\
\text { em usuários habituais } \\
\text { ou dependentes }\end{array}$ & $\begin{array}{l}\text { Verificou-se que a } \\
\text { deficiência de } \\
\text { suporte parental, a } \\
\text { superproteção dos } \\
\text { filhos, a presença de } \\
\text { cultura implícita do } \\
\text { uso de drogas, a } \\
\text { existência r de } \\
\text { conflitos r } \\
\text { violências, } \\
\text { desinformação e } \\
\text { desconhecimento } \\
\text { sobre o uso de } \\
\text { drogas a } \\
\text { influenciaram o uso } \\
\text { de crack entre } \\
\text { familiares de } \\
\text { dependentes dessa } \\
\text { droga. }\end{array}$ \\
\hline $\begin{array}{l}\text { The association } \\
\text { between parental } \\
\text { risk behaviors } \\
\text { during childhood } \\
\text { and having high } \\
\text { risk networks in } \\
\text { adulthood (Nova } \\
\text { Iorque-EUA, } \\
\text { 2011) }\end{array}$ & $\begin{array}{lr}650 & \text { jovens } \\
\text { usuários } & \text { de } \\
\text { drogas com média } \\
\text { de idade de } 33 \\
\text { anos }\end{array}$ & $\begin{array}{l}\text { Coleta de dados } \\
\text { em intervenções } \\
\text { de rua na cidade } \\
\text { de Nova Iorque } \\
\text { (EUA) }\end{array}$ & $\begin{array}{l}\text { Longitudinal } \\
\text { (Qualitativo- } \\
\text { entrevistas) } \\
\text { (9/10 CASP } \\
\text { Qualitative } \\
\text { Research } \\
\text { Checklist) }\end{array}$ & $\begin{array}{l}\text { Avaliar a associação } \\
\text { entre o uso de drogas } \\
\text { por mãe, pai ou } \\
\text { cuidador primário } \\
\text { durante a infância e ter } \\
\text { uma maior chance de } \\
\text { usar drogas na idade } \\
\text { adulta }\end{array}$ & $\begin{array}{l}\text { Vinte e dois por } \\
\text { cento dos } \\
\text { participantes usavam } \\
\text { drogas injetáveis, } \\
49 \% \text { eram negros, e } \\
\text { durante a infância } \\
26 \% \text { das mães, 32\% } \\
\text { dos pais, e 13\% dos } \\
\text { primeiros cuidadores } \\
\text { usavam drogas. Ter } \\
>1 \text { familiar que usou } \\
\text { drogas foi associado } \\
\text { a ter uma grande } \\
\text { probabilidade de } \\
\text { usar drogas (IC= } \\
95 \% \text { e } \\
\text { especificamente o } \\
\text { uso/fumo do crack } \\
\text { em seus ambientes } \\
\text { na fase adulta. }\end{array}$ \\
\hline
\end{tabular}




\begin{tabular}{|c|c|c|c|c|c|}
\hline $\begin{array}{lr}\begin{array}{l}\text { Individual } \\
\text { factors }\end{array} & \begin{array}{r}\text { risk } \\
\text { for }\end{array} \\
\text { adolescent } & \\
\text { substance } & \text { use, } \\
\text { (Emirados Árabes } & \text { Unidos, 1999) }\end{array}$ & $\begin{array}{l}\text { Adolescentes } \\
\text { (população alvo) }\end{array}$ & Não se aplica & $\begin{array}{l}\text { Revisão } \\
\text { sistemática }(2 / 11 \\
\text { AMSTAR) }\end{array}$ & $\begin{array}{l}\text { Identificar os fatores de } \\
\text { risco e estabelecer } \\
\text { estratégia de prevenção } \\
\text { para o uso de drogas } \\
\text { em adolescentes }\end{array}$ & $\begin{array}{l}\text { O uso de substâncias } \\
\text { ilícitas pelos pais } \\
\text { claramente } \\
\text { influencia no } \\
\text { comportamento de } \\
\text { risco dos filhos, } \\
\text { principalmente na } \\
\text { escolha de um grupo } \\
\text { de comportamento } \\
\text { de risco como } \\
\text { modelo de amizade. } \\
\text { Vários fatores } \\
\text { podem influenciar } \\
\text { no início do uso de } \\
\text { drogas como a carga } \\
\text { genética herdada dos } \\
\text { pais, a falta e/ou o } \\
\text { excesso de controle } \\
\text { exercido pelos pais, } \\
\text { bem como a } \\
\text { influência dos } \\
\text { grupos sociais e } \\
\text { também do meio } \\
\text { onde os adolescentes } \\
\text { estão inseridos. }\end{array}$ \\
\hline $\begin{array}{l}\text { Abuso } \\
\text { dependência: } \\
\text { crack } \\
(\text { Brasil, 2012) }\end{array}$ & Não se aplica & Não se aplica & $\begin{array}{l}\text { Revisão } \\
\text { sistemática (4/11 } \\
\text { AMSTAR) }\end{array}$ & $\begin{array}{l}\text { Atualizar sobre as } \\
\text { especificidades na } \\
\text { detecção precoce e na } \\
\text { abordagem do usuário } \\
\text { de crack }\end{array}$ & 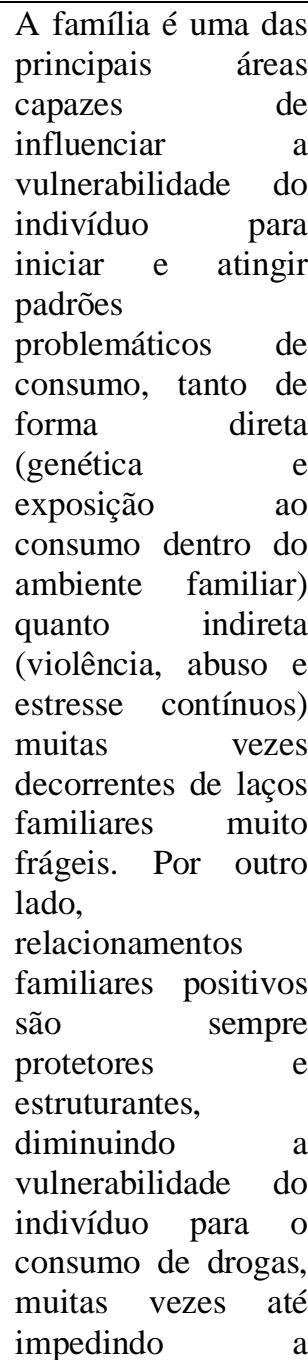 \\
\hline
\end{tabular}




\begin{tabular}{|c|c|c|c|c|c|}
\hline & & & & & $\begin{array}{l}\text { dependência } \quad \text { de } \\
\text { determinada } \\
\text { substância. }\end{array}$ \\
\hline $\begin{array}{l}\text { Filhos de } \\
\text { dependentes } \\
\text { químicos com } \\
\text { fatores de risco } \\
\text { bio-psicossociais: } \\
\text { necessitam de um } \\
\text { olhar especial? } \\
(\text { São Paulo-Brasil, } \\
2004)^{4}\end{array}$ & $\begin{array}{ll}63 \text { familiares } & \text { de } \\
\text { dependentes } & \\
\text { químicos, } & 54 \\
\text { crianças e } & 45 \\
\text { adolescentes } & \end{array}$ & $\begin{array}{l}\text { Coleta de dados } \\
\text { no Serviço de } \\
\text { Prevenção } \\
\text { Seletiva na } \\
\text { periferia de São } \\
\text { Paulo }\end{array}$ & $\begin{array}{l}\text { Transversal }(9 / 11 \\
\text { Cross Sectional } \\
\text { Appraisal Tool) }\end{array}$ & $\begin{array}{l}\text { Investigar o perfil de } \\
\text { crianças, adolescentes e } \\
\text { familiares em um } \\
\text { serviço de prevenção } \\
\text { seletiva para filhos de } \\
\text { dependentes químicos e } \\
\text { discutir alternativas de } \\
\text { intervenção } \\
\text { tratamento para essa } \\
\text { população }\end{array}$ & $\begin{array}{l}\text { Na maioria das } \\
\text { famílias o pai é o } \\
\text { dependente químico } \\
(67 \%) \text {, tendo como } \\
\text { substância de } \\
\text { escolha o álcool } \\
(75 \%) \text {. Detectou-se } \\
\text { que, em 59\% dos } \\
\text { cônjuges que não } \\
\text { eram dependentes } \\
\text { químicos, risco de } \\
\text { distúrbios em saúde } \\
\text { mental. } \\
\text { crianças, Nas } \\
\text { observada timidez e } \\
\text { sentimento de } \\
\text { inferioridade, } \\
\text { depressão, conflito } \\
\text { familiar, carência } \\
\text { afetiva e de bom } \\
\text { nível de energia, que } \\
\text { é indicativo de } \\
\text { desequilíbrio } \\
\text { emocional e mental. } \\
\text { Nos adolescentes, } \\
\text { foi observado maior } \\
\text { índice de problemas } \\
\text { nas seguintes áreas } \\
\text { do DUSI: desordens } \\
\text { psiquiátricas, } \\
\text { sociabilidade, famiar e } \\
\text { sistema familiar } \\
\text { lazer/ Recreação. }\end{array}$ \\
\hline $\begin{array}{l}\text { Children of } \\
\text { African- } \\
\text { American } \\
\text { Mothers who use } \\
\text { crack cocaine: } \\
\text { parenting } \\
\text { influences on } \\
\text { youth substance } \\
\text { use (Carolina do } \\
\text { Norte-EUA, } \\
\text { 2007) }\end{array}$ & $\begin{array}{l}208 \text { pares mãe- } \\
\text { filho, sendo que } \\
\text { as mães tinham } \\
\text { idade entre } 14-36 \\
\text { anos }\end{array}$ & $\begin{array}{lr}\text { As mães foram } \\
\text { recrutadas } \\
\text { ruas, em } \\
\text { onde casas } \\
\text { usuários } & \text { vivem } \\
\text { crack e } & \text { de } \\
\text { locais } & \text { de } \\
\text { encontro para o } \\
\text { uso do crack na } \\
\text { Carolina } \\
\text { Norte. }\end{array}$ & $\begin{array}{l}\text { Transversal }(8 / 10 \\
\text { Cross Sectional } \\
\text { Appraisal Tool) }\end{array}$ & $\begin{array}{l}\text { Examinar as relações } \\
\text { entre mães usuárias de } \\
\text { crack e seus filhos; e } \\
\text { avaliar os efeitos dessa } \\
\text { relação no início } \\
\text { precoce do abuso de } \\
\text { substâncias }\end{array}$ & 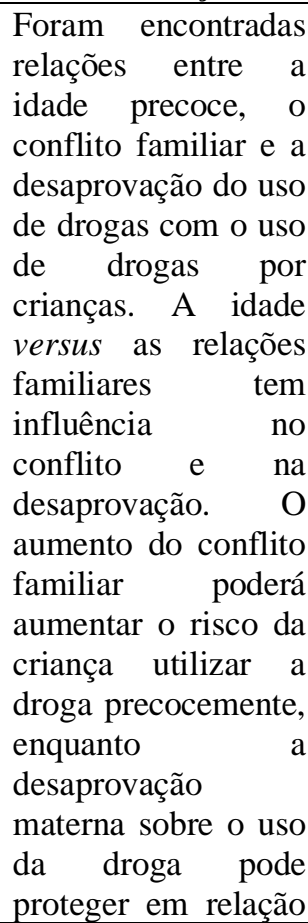 \\
\hline
\end{tabular}




\begin{tabular}{|c|c|c|c|c|c|}
\hline & & & & & ao uso precoce. \\
\hline \begin{tabular}{lr}
\multicolumn{3}{l}{ Razões para o não } \\
uso de drogas \\
ilícitas \\
jovens entre \\
situação de risco \\
$($ Brasil, 2005)
\end{tabular} & $\begin{array}{l}62 \text { jovens de } \\
\text { ambos os sexos } \\
\text { com idade entre } \\
16 \text { e } 24 \text { anos, de } \\
\text { classe rocial } \\
\text { baixa, sendo que } \\
32 \text { deles nunca } \\
\text { usaram drogas } \\
\text { ilícitas e } 30 \\
\text { sujeitos fizeram } \\
\text { uso pesado }\end{array}$ & $\begin{array}{l}\text { Os jovens foram } \\
\text { recrutados por } \\
\text { meio da técnica } \\
\text { de bola-de-neve, } \\
\text { onde os } \\
\text { primeiros on } \\
\text { entrevistados } \\
\text { indicam outros, } \\
\text { que por sua vez } \\
\text { indicam outros e } \\
\text { assim haviam } \\
\text { sucessivamente, } \\
\text { não harticipantes } \\
\text { partificados } \\
\text { classifical } \\
\text { quanto o local } \\
\text { de moradia } \\
\text { (bairro), escola, } \\
\text { amigos } \\
\text { comunidade } \\
\text { religiosa. }\end{array}$ & $\begin{array}{l}\text { Qualitativo (9/10 } \\
\text { CASP } \\
\text { Qualitative } \\
\text { Research } \\
\text { Checklist) }\end{array}$ & $\begin{array}{l}\text { Identificar, entre } \\
\text { adolescentes de baixo } \\
\text { poder aquisitivo, quais } \\
\text { os motivos que os } \\
\text { impediriam a } \\
\text { experimentação e o } \\
\text { consequente uso de } \\
\text { drogas psicotrópicas, } \\
\text { mesmo quando } \\
\text { submetidos a constante } \\
\text { oferta }\end{array}$ & 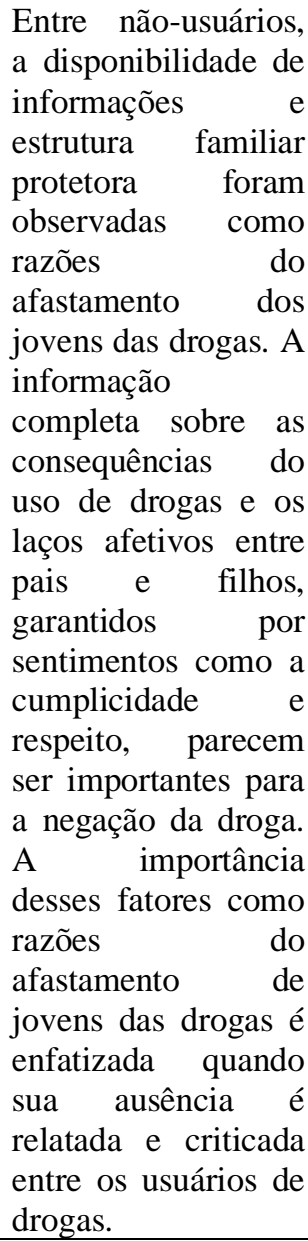 \\
\hline $\begin{array}{l}\text { Prenatal and } \\
\text { postnatal cocaine } \\
\text { exposure predict } \\
\text { teen cocaine use } \\
(\text { Michigan-EUA, } \\
2011)^{12}\end{array}$ & $\begin{array}{l}\text { Adolescentes } \\
\text { afro-americanos } \\
\text { incialmente } \\
\text { identificados por } \\
\text { um estudo } \\
\text { prospectivo da } \\
\text { gravidez. }\end{array}$ & \begin{tabular}{l}
\multicolumn{2}{l}{ Adolescentes } \\
recrutados por \\
estudo anterior \\
realizado pelo \\
centro \\
universidade/ma \\
ternidade do \\
interior da \\
cidade de \\
Detroit, \\
posteriormente \\
entrevistados \\
assim como \\
suas mães.
\end{tabular} & \begin{tabular}{ll}
\multicolumn{2}{l|}{ Coorte } \\
prospectivo \\
$(10 / 12$ & CASP \\
Cohort & Study \\
Checklist) &
\end{tabular} & $\begin{array}{l}\text { Avaliar a relação entre } \\
\text { a exposição pré-natal à } \\
\text { cocaína e o uso de } \\
\text { cocaína } \\
\text { adolescentes }\end{array}$ & 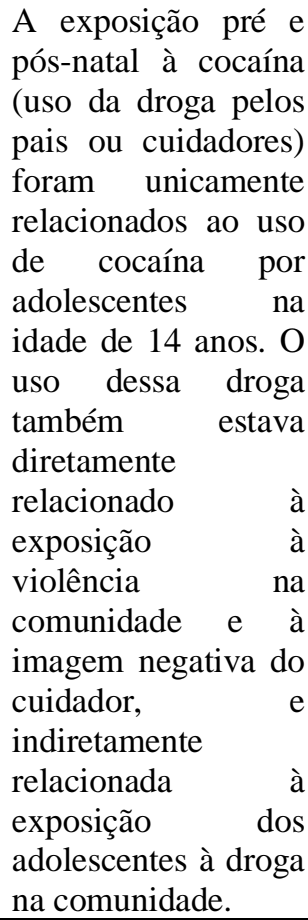 \\
\hline
\end{tabular}




\begin{tabular}{|c|c|c|c|c|c|}
\hline $\begin{array}{l}\text { Fatores de risco } \\
\text { para o consumo } \\
\text { de crack na } \\
\text { adolescência (Rio } \\
\text { Grande do Sul- } \\
\text { Brasil, 2011) }{ }^{10}\end{array}$ & $\begin{array}{l}\text { Adolescentes até } \\
21 \text { anos de idade } \\
\text { incompletos, } \\
\text { usuários de crack } \\
\text { que frequentavam } \\
\text { serviços de saúde } \\
\text { em Santa Cruz do } \\
\text { Sul (Brasil) }\end{array}$ & $\begin{array}{l}9 \text { entrevistas } \\
\text { retiradas de uma } \\
\text { pesquisa de } \\
\text { abrangência } \\
\text { municipal, } \\
\text { intitulada de "A } \\
\text { realidade do } \\
\text { crack em Santa } \\
\text { Cruz do Sul" no } \\
\text { ano de } 2010\end{array}$ & $\begin{array}{l}\text { Quantitativo } \\
\text { (7/12 McMaster } \\
\text { Quantitative } \\
\text { review) }\end{array}$ & $\begin{array}{l}\text { Investigar os fatores de } \\
\text { risco no consumo de } \\
\text { crack na adolescência }\end{array}$ & 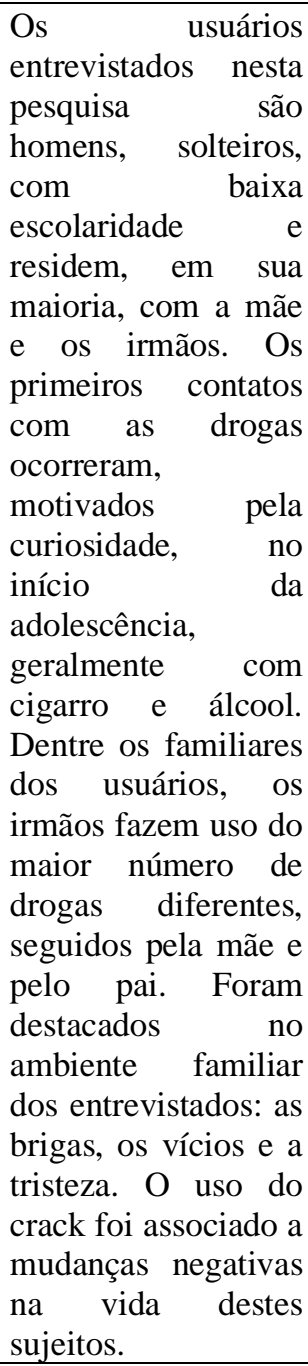 \\
\hline $\begin{array}{l}\text { A importância da } \\
\text { família na } \\
\text { prevenção do uso } \\
\text { de drogas entre } \\
\text { crianças } \\
\text { adolescentes: } \\
\text { papel materno } \\
(\text { Rio de Janeiro- } \\
\text { Brasil, 2008) }\end{array}$ & $\begin{array}{l}22 \text { mães (maiores } \\
\text { de } 18 \text { anos) de } \\
\text { uma comunidade } \\
\text { situada no } \\
\text { município do Rio } \\
\text { de Janeiro }\end{array}$ & $\begin{array}{l}\text { Entrevistas } \\
\text { realizadas, no } \\
\text { ano de 2006, } \\
\text { com as mães no } \\
\text { momento em } \\
\text { que iam buscar } \\
\text { seus filhos na } \\
\text { creche da } \\
\text { comunidade }\end{array}$ & $\begin{array}{l}\text { Qualitativo } \\
\text { descritivo } \quad(7 / 10 \\
\text { CASP } \\
\text { Qualitative } \\
\text { Research } \\
\text { Checklist) }\end{array}$ & $\begin{array}{l}\text { Descrever os fatores de } \\
\text { risco e os fatores } \\
\text { protetores para o uso e } \\
\text { abuso de drogas } \\
\text { referidas pela família }\end{array}$ & $\begin{array}{l}\text { As mães relataram } \\
\text { como fatores de } \\
\text { risco o meio, a } \\
\text { influência dos pares } \\
\text { e a utilização de } \\
\text { drogas pela família. } \\
\text { Como fatores } \\
\text { protetores o diálogo, } \\
\text { a família estruturada } \\
\text { e atividades sociais e } \\
\text { educativas. }\end{array}$ \\
\hline
\end{tabular}

\section{DISCUSSÃO}

A dependência química, principalmente de substâncias com alto índice de vício, como o crack, tende a desestabilizar a família do usuário dessa droga. Ademais, com o crescimento do uso dessa substância no Brasil, é importante verificar os fatores que predispõem os indivíduos a se tornarem dependentes dessa droga, bem como as razões para não usá-la. A heterogeneidade das intervenções, tipos de estudos e seus desfechos dificultaram o agrupamento e a comparação dos achados dos estudos 
incluídos, contudo pode-se perceber que o ambiente familiar e social é um fator protetor e de risco para o início ao uso de drogas, principalmente o crack.

De acordo com o estudo qualitativo recente e de alta qualidade metodológica, os vínculos familiares deficientes, podem tanto estimular o início da dependência quanto a manutenção do uso de $\mathrm{crack}^{5}$. Além disso, esse estudo afirma que a falta de informação e desconhecimento sobre o uso de drogas também foram fatores que influenciaram familiares de dependentes iniciarem o uso. Corroborando esse achado, um estudo longitudinal recente e de alta qualidade metodológica, demonstra a associação entre a existência de mais de um familiar usuário de drogas com uma grande probabilidade dos filhos também usarem drogas $(\mathrm{IC}=95 \%)$ e, mais especificamente, o uso/fumo do crack em seus ambientes na fase adulta. Nesse ambiente favorável ao futuro consumo de crack, 26\% das mães usaram crack durante a infância desse provável usuário de crack ${ }^{7}$.

Afirmando as conclusões supracitadas, um estudo qualitativo descritivo de alta qualidade metodológica foi realizado, baseado principalmente na opinião das mães sobre os fatores de risco e protetores para o uso de drogas que circundam seus filhos. Concluiu-se com o estudo que as mães têm consciência que a influência familiar e social é o principal fator que poderá levar seus filhos ao uso de drogas. Além disso, elas afirmam que o diálogo, uma família estruturada e atividades sociais e educativas podem atuar como fatores protetores ao uso e abuso de drogas pelos jovens ${ }^{9}$.

Essa influência materna em relação ao uso de crack pelos seus filhos é confirmada pela pesquisa transversal recente e de alta qualidade metodológica, que demonstra como a influência no comportamento da criança é de forma significativa e mais fortemente imposta pelas mães do que pelos pais ${ }^{6}$. Portanto, a mãe ser usuária de crack, pode afetar de forma negativa o comportamento dos filhos, elevando o risco dessas crianças se tornarem usuárias de crack quando jovens e adultos. Além disso, o estudo também concluiu que o aumento do conflito familiar pode aumentar substancialmente o risco do jovem se tornar um usuário de drogas, em contrapartida, a desaprovação da mãe especificamente pode atuar como fator protetor ao consumo de drogas ${ }^{6}$. Assim como concluiu uma revisão sistemática antiga e de baixa qualidade metodológica, na qual há a afirmação de que há influência do ambiente, incluindo familiares e amigos, no uso precoce de drogas ${ }^{8}$.

Concluiu-se também em estudo transversal recente de alta qualidade metodológica que existe realmente maior probabilidade da criança/jovem tornar-se usuário de drogas quando há no convívio familiar esse consumo. Contudo, nesse estudo foi encontrado que o pai seria o dependente químico $(67 \%)$, sendo que $59 \%$ das mães ou cônjuges não eram dependentes ${ }^{4}$. Outro estudo que corrobora essa ideia, em que foi feita uma pesquisa qualitativa de média qualidade metodológica, no qual concluiu-se que a influência do ambiente familiar foi predominante em relação aos outros fatores predisponentes ao 
uso de crack pelos entrevistados, sendo que na maioria dos casos, pelo menos um familiar fazia uso de algum tipo de droga, e foi constatado que as mudanças negativas na vida do sujeito estão intimamente relacionados com o uso de $\mathrm{crack}^{10}$.

Já no estudo qualitativo com alta qualidade metodológica, não foram pesquisados apenas os fatores de risco, mas também os fatores protetores ao uso de crack, abordando com maior relevância esse aspecto. Os fatores de risco encontrados foram aqueles supracitados. Já em relação aos fatores protetores ao uso precoce da droga, concluiu-se que podem ser: a boa relação familiar, a desaprovação do meio social à droga, o interesse familiar na vida da criança e/ou adolescente além da realização de atividades socioeducativas no ambiente em que esses jovens estavam inseridos ${ }^{11}$. Outros estudos analisados também abordam os fatores protetores ao uso precoce de droga/crack, sendo a conclusão final destes concordante com aquela que foi apresentada no estudo anteriormente analisado ${ }^{6,8,9}$.

Além dos fatores de risco e protetores já citados que foram unanimidade na maioria dos estudos, uma revisão sistemática recente de baixa qualidade metodológica, relatou a existência da herdabilidade de forma direta, ou seja, há influência genética para o abuso e dependência de $\operatorname{crack}^{13}$. Por fim, foi encontrada relação entre a exposição pré-natal com o uso de cocaína por adolescentes, em um estudo do tipo coorte retrospectivo de alta qualidade metodológica, além da relação pré-natal há a evidência da exposição pós-natal que está relacionada intimamente com a influência do ambiente familiar e social citada na maioria dos artigos analisados. Nesse caso há a ênfase no aspecto do contato na comunidade ${ }^{12}$.

\section{LIMITAÇÕES}

Esta revisão sistemática apresenta como principais limitações o número de bases de dados utilizadas; alguns fatos terem sido relatados por terceiros; e o fato de que alguns estudos exigiram dos participantes resgatar memórias antigas da fase de infância. A primeira ocorreu devido a limitação de tempo para a execução da pesquisa, que fez com que houvesse apenas a utilização de três bancos de dados. Possivelmente a inclusão de outras bases como PsycINFO poderia ter enriquecido a recuperação de artigos relevantes.

Já a segunda, relaciona-se à existência de viés de memória nos estudos que dependeram de relato de filhos e/ou cuidadores referentes ao uso de crack durante a gestação e puerpério. Sendo que a terceira foi devido à possibilidade de que as memórias antigas da fase de infância fossem distorcidas. No entanto, isso não mudaria o tipo de droga que foi usada na pesquisa. 


\section{CONCLUSÃO}

A análise do presente estudo, baseado na revisão sistemática através dos artigos selecionados acima teve por finalidade avaliar os efeitos do uso de crack antes, durante e após a gestação e sua implicação no desenvolvimento da dependência da droga futuramente. Além disso, propôs identificar quais os fatores influenciaram no uso do crack por filhos de mães dependentes e quais fatores foram classificados como protetores para esse uso.

Com isso, foi constatado que a herdabilidade dos vícios e a exposição pré-natal podem influenciar no futuro uso dessa droga por filhos de usuários, contudo, não foram encontrados muitos estudos que corroborassem essa afirmativa. Já a exposição pós-natal, seja ela feita por familiares ou amigos, podem influenciar no futuro uso dessa droga, visto que, há uma maior probabilidade de crianças que convivem com familiares usuários de droga se tornem futuramente também usuários de droga.

Conclui-se então, que filhos de dependentes químicos representam um grupo de risco para o futuro uso de drogas, mais especificamente o crack. Mas que a relação do uso durante a gestação não está clara. Dessa forma, aumenta a importância e a necessidade de um serviço preventivo especializado dirigido às crianças, adolescentes, jovens e familiares de dependentes químicos, principalmente em uso e abuso do crack. 


\section{REFERÊNCIAS}

1. Zero Hora. Número de usuários de crack no país ultrapassa 1 milhão, diz especialista. 2010. [acesso em 02 out 2013]. Disponível em: http://zerohora.clicrbs.com.br/rs/geral/noticia/2010/05/numerode-usuarios-de-crack-no-pais-ultrapassa-1-milhao-diz-especialista-2894303.html

2. Fundação Oswaldo Cruz. Maior pesquisa sobre crack já feita no mundo mostra o perfil do consumo no Brasil. 2013. [acesso em 28 set 2013]. Disponível em: http://portal.fiocruz.br/pt-br/content/maiorpesquisa-sobre-crack-j\%C3\%A1-feita-no-mundo-mostra-o-perfil-do-consumo-no-brasil

3. Gasparin M, Silveira JL, Garcez LW, Levy BS. Comportamento motor oral e global de recémnascidos de mães usuárias de crack e/ou cocaína. Revista da Sociedade Brasileira de Fonoaudiologia. 2012 dez.; 17(4): 459-463 Available from: http://www.scielo.br/scielo.php?script=sci_arttext\&pid=S1516-80342012000400016\&lng=en\&nrm=iso

4. Figlie N, Fontes A, Moraes E, Payá R. Filhos de dependentes químicos com fatores de risco biopsicossociais: necessitam de um olhar especial?. Revista de Psiquiatria Clínica. 2004; n. 31(2): 53-62.

5. Seleghim MR, Magda LFO. Influência do ambiente familiar no consumo de crack em usuários. Acta Paulista de Enfermagem. 2013 jun.; 26(3): 263-8 Available from: http://www.scielo.br/scielo.php?script=sci_arttext\&pid=S0103-

$21002013000300010 \& \operatorname{lng}=$ en \&nrm=iso

6. Lam WKK, Cance JD, Eke AN, Fishbein DH, Hawkins SR, Williams JC. Children of AfricanAmerican Mothers Who Use Crack Cocaine: Parenting Influences on Youth Substance Use. Journal of Pediatric Psychology. 2007 mai; 32(8): 877-887

7. Rudolph AE, Jones KC, Latkin C, Crawford ND, Fuller CM. The association between parental risk behaviors during childhood and having high risk networks in adulthood. Drug Alcohol Dependence. 2011 nov; 118(2-3): 437-443.

8. Swadi H. Individual risk factors for adolescent substance use. Drug and Alcohol Dependence. 1999; 55: 209-224.

9. Oliveira EB, Bittencourt LB, Carmo AC. A importância da família na prevenção do uso de drogas entre crianças e adolescentes: papel materno. Revista Eletrônica Saúde Mental e Drogas. 2008; 4(2).

10. Santos EMAL, Silva JC, Linhares EG, Zacharias DG. Fatores de risco para o consumo de crack na adolescência. IV Jornada de Pesquisa em Psicologia. 2011.

11. ZILA Sanchez ZM, Oliveira LG, Nappo SA. Razões para o não-uso de drogas ilícitas entre jovens em situação de risco. Revista de Saúde Pública. 2005; 39(4): 599-605.

12. Delaney-Black V, Chiodo LM, Hannigan JG, Greenwald MK, Janisse J, Patterson G, Huestis MA, Partridge RT, Ager J, Sokol RJ. Prenatal and postnatal cocaine exposure predict teen cocaine use. Neurotoxicology and Teratology. 2011; 33: 110-119.

13. Marques ACPR, Ribeiro M, Laranjeira RR, Andrada NC. Abuso e dependência: crack. Rev. Assoc. Med. Bras.; 58(2). Available from: http://www.scielo.br/scielo.php?pid=S010442302012000200008\&script=sci_arttext. 
14. Critical Appraisal Skills Programe: Making Sense of Evidence [Internet].Oxford(UK).1993 [citado em out/2013]. Disponível em: http://www.casp-uk.net.

15. Shea BJ, Hamel C, Wells GA, Bouter LM, Kristjansson E, Grimshaw J, Henry DA, Boers M. AMSTAR is a reliable and valid measurement tool to assess the methodological quality of systematic reviews. J Clin Epidemiol. 2009 Oct; 62(10): 1013-20.

16. Critical Appraisal Tools. [Internet] Canadá: Universidade McMaster. 1998. [citado em Out/2013]. Disponível em: http://www.srs-mcmaster.ca/Portals/20/pdf/epb/quanreview.pdf.

17. Niccols A, Milligan K, Smith A, Sword W, Thabane L, Henderson J. Integrated programs formothers withsubstance abuse issues and their children: A systematic review of studies reporting on child outcomes. Child Abuse \& Neglec. 2012 abr; 36: 308-322. 